\title{
Turbo-Coded Adaptive Modulation Versus Space-Time Trellis Codes for Transmission Over Dispersive Channels
}

\author{
T. H. Liew, Bee Leong Yeap, Choong Hin Wong, and Lajos Hanzo, Fellow, IEEE
}

\begin{abstract}
Decision feedback equalizer (DFE)-aided turbocoded wideband adaptive quadrature amplitude modulation (AQAM) is proposed, which is capable of combating the temporal channel quality variation of fading channels. A procedure is suggested for determining the AQAM switching thresholds and the specific turbo-coding rates capable of maintaining the target bit-error rate while aiming for achieving a highly effective bits per symbol throughput. As a design alternative, we also employ multiple-input/multiple-output DFE-aided space-time trellis codes, which benefit from transmit diversity and hence reduce the temporal channel quality fluctuations. The performance of both systems is characterized and compared when communicating over the COST 207 typical urban wideband fading channel. It was found that the turbo-coded AQAM scheme outperforms the two-transmitter space-time trellis coded system employing two receivers; although, its performance is inferior to the space-time trellis coded arrangement employing three receivers.
\end{abstract}

Index Terms-Coded adaptive modulation, dispersive channels, space-time trellis codes.

\section{INTRODUCTION}

$\mathbf{I}$ N HIS seminal contribution on adaptive transceivers Hayes [1] proposed transmission power adaptation, while Cavers [2] advocated the employment of a variable symbol duration scheme. Hayes' method inflicts increased cochannel interference upon other users, while Cavers' technique has a variable bitrate or a variable bandwidth requirement. Adaptive quadrature amplitude modulation (AQAM) was proposed by Steele and Webb as a design alternative, employing various differentially encoded star-QAM constellations [3]. As a further advance, Otsuki et al. [4] employed coherently detected pilot symbol assisted modulation (PSAM)-aided square constellations instead of differentially detected star constellations for AQAM. Goldsmith and Varaiya [5] demonstrated that variable-power variable-rate adaptive schemes are optimum in terms of approaching the capacity of nondispersive Rayleigh fading channels [6] and they quantified the average throughput of variable-power AQAM [5]. However, Goldsmith and Varaiya also found that the extra channel capacity achieved by variable-power assisted adaptation over the constant-power variable-rate scheme is a fraction of a decibel for most fading channels [5], [7].

Manuscript received December 16, 2001; accepted June 13, 2003. The editor coordinating the review of this paper and approving it for publication is C. Tellambura.

The authors are with the School of ECS, University of Southampton, Southampton SO17 1BJ, U.K. (e-mail: 1h@ecs.soton.ac.uk)

Digital Object Identifier 10.1109/TWC.2004.837424
The achievable effective throughput and bit-error rate (BER) of AQAM modems is critically dependent on the choice of the AQAM mode switching levels. Torrance and Hanzo introduced the average BER of AQAM experienced over fading channels as the optimization constraint and optimized the switching levels for achieving as high an average throughput as possible [8] with the aid of Powell's optimization [9]. Since the resultant average BER showed a good agreement with the target average BER, Torrance's switching levels have been used for numerous simulation studies [10]-[14]. However, since these switching levels are constant across the entire range of signal-to-noise (SNR) values, the average BER varies slightly and the SNR range over which the average BER remains constant is limited. Recently, Choi et al. proposed a new set of SNR-dependent switching levels optimized at each SNR value in order to maximize the average throughput while maintaining the target average BER up to the avalanche SNR point, beyond which the highest order constituent modulation mode is activated [15]. The set of optimum switching levels derived using a Lagrangian multiplier-based optimization [16] technique was documented in more detail in [17] and [18].

Based on this seminal research, adaptive trasnceivers have found their way into oprational wireless systems [19] such as the high-speed data packet access mode of the $3 \mathrm{G}$ systems [20]. AQAM was also invoked in the context of orthogonal frequency-division multiplex (OFDM) [13], [21], code-division multiple access (CDMA) [11], [12], [22], [23], and radial basis function (RBF)-aided nonlinear transceivers [24].

A range of adaptive trellis-coded modulation (TCM) schemes has been investigated in the literature for transmission over narrowband fading channels [25]-[28]. In particular, adaptive trellis-coded M-ary PSK was considered in [25] and coset codes were applied to adaptive trellis-coded M-ary QAM in [26]. However, these results were based on idealized assumptions, such as perfect channel estimation and zero feedback delay. In [27], adaptive TCM using outdated fading estimates was investigated. Recently, the performance of adaptive TCM based on practical considerations such as channel estimation noise, feedback noise, and feedback delay was evaluated in [28]. It was found that the adaptive TCM scheme is robust in most practical situations. A range of practically motivated wideband AQAM TCM, turbo TCM (TTCM), bit-interleaved coded modulation (BICM) and iteratively decoded BICM (BICM-ID) were investigated in [29], while their application to parallel interference cancellation (PIC)-aided CDMA systems was considered in [23]. 
The performance of both space-time trellis and block codes was investigated by numerous researchers [30]-[34] when communicating over narrowband Rayleigh fading channels. The investigation of space-time codes was then also extended to the class of more practical wideband fading channels. The effect of multiple paths on the performance of space-time trellis codes was studied in [35] for transmission over slowly varying Rayleigh fading channels. It was shown in [35] that the presence of multiple paths does not decrease the diversity order guaranteed by the design criteria used for constructing space-time trellis codes. The evidence provided in [35] was then also extended to rapidly fading dispersive and nondispersive channels. As a further performance improvement, turbo equalization was employed in [36] in order to mitigate the effects of dispersive channels. As an alternative solution, the effect of intersymbol interference (ISI) could also be eliminated by employing orthogonal frequency-division multiplexing (OFDM) [37]. A system using space-time trellis-coded OFDM is attractive, since the decoding complexity can be reduced, as demonstrated by the recent surge of research interests [32], [38]-[40]. Similarly, the performance of space-time block codes was also investigated when communicating over frequency selective Rayleigh fading channels. In [41], a multiple-input/multiple-output (MIMO) equalizer was utilized for equalizing the effects of dispersive multipath channels. In this contribution, the MIMO equalizer was employed in conjunction with space-time trellis codes for equalizing the wideband channel. The minimum mean-squared error (MMSE) MIMO equalizer was first studied by Duel-Hallen [42] in the transform domain. It was then employed by Tidestav et al. in [43] for multiuser detection. In space-time trellis (STT) codecs there are multiple transmitters and receivers at both the transmitting and receiving ends. The received symbol at each receiver is constituted by the superposition of all transmitted symbols, which undergo independent wideband fading. The MIMO equalizer was then employed for equalizing and estimating the transmitted symbols of each transmitter.

In [44], we have shown that as we increase the diversity order of STT coding, the fluctuation of channel quality becomes less severe, since the fading channels have been converted to AWGN-like channels. By contrast, AQAM seeks to accommodate the temporal variation of the quality of fading channels by appropriately adjusting the turbo-coded AQAM modes. Therefore, in this contribution, we will investigate the achievable throughput of adaptive coded AQAM and space-time trellis codes. In Section II, we provide a brief system overview, while in Section III the simulation parameters are summarized. Section IV is dedicated to performance discussions, before we offer our conclusions in Section V.

\section{SYSTEM OVERVIEW}

Fig. 1 shows the system overview of the proposed turbo-coded AQAM scheme as well as the space-time trellis codes employed. At the transmitter, the information source generates random information data bits. In our proposed turbo-coded AQAM scheme, the information data bits are

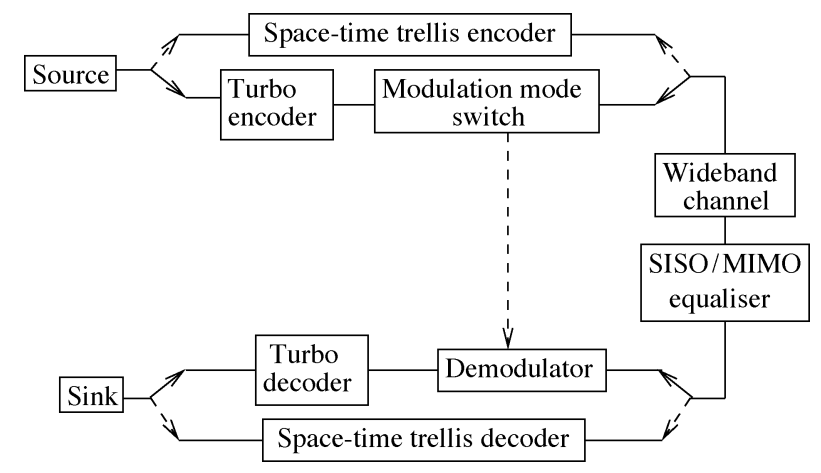

Fig. 1. System overview of the turbo-coded AQAM and space-time trellis schemes.

encoded by the turbo encoder. Assuming that the next transmission burst's short term channel quality can be sufficiently accurately estimated [18] by the receiver, a suitable AQAM mode will be chosen by the modulation mode switch shown in the figure. The turbo-coded bits are subsequently mapped to the AQAM symbols. By contrast, the information bits will be directly encoded by the STT encoder, which produces the modulated symbols depending on the specific STT code employed. The modulated symbols are transmitted over the COST $207 \mathrm{TU}$ wideband channel. At the receiver, the received symbols are equalized by the appropriate single-input/single-output (SISO) or MIMO DFE, respectively. Depending on the specific AQAM mode chosen at the transmitter, the demodulator provides soft outputs for the turbo decoder based on the equalized symbols output by the SISO equalizer. The turbo decoded bits are passed to the information sink for the calculation of the BER, as shown in Fig. 1. By contrast, the equalized symbols output by the MIMO equalizer are passed to the space-time trellis decoder.

\section{A. SISO Equalizer and AQAM}

At the receiver, the channel impulse response (CIR) is estimated, which is then used for calculating the SISO DFE coefficients [37]. Subsequently, the DFE is used for equalizing the ISI-corrupted received signal. Additionally, both the channel quality estimate and the SISO DFE coefficients are utilized for computing the pseudo-SNR at the output of the SISO DFE. By assuming that the residual ISI is Gaussian distributed and that the probability of decision feedback errors is negligible, the pseudo-SNR at the output of the SISO DFE $\gamma_{\mathrm{dfe}}$ can be calculated as [45] in (1), shown at the bottom of the next page, where $C_{m}$ and $h_{m}$ denote the DFE's feedforward coefficients and the CIR, respectively. The transmitted signal and the noise spectral density are represented by $S_{k}$ and $N_{o}$, respectively. Lastly, the number of DFE feedforward coefficients is denoted by $N_{f}$.

The pseudo-SNR $\gamma_{\mathrm{dfe}}$ is then compared against a set of AQAM switching threshold levels $f_{n}$, and subsequently the appropriate modulation mode is selected for the next transmission burst, assuming the availability of a reliable low-delay feedback message, which can be transmitted to the remote receiver by superimposing it (after strongly protecting it) on the reverse-direction information [10]. However, in this treatise we will dispense with explicit AQAM mode signaling. The AQAM modes that are utilized in this system are binary phase 
shift keying (BPSK), quadrature phase shift keying (QPSK), 16-level quadrature amplitude modulation (16QAM), 64-level quadrature amplitude modulation (64QAM) and a "no transmission" (NO TX) mode.

\section{B. MIMO Equalizer}

In the context of the MIMO equalizer, similarly to the AQAM receiver, the CIR is estimated, which is then used for calculating the MIMO DFE coefficients [43]. At receiver $R x$, the received signal is given by the superposition of all the transmitters' signals. For each channel delay $m$ and each combination of the specific transmitters and receivers of the MIMO scheme, we have a specific CIR. Hence, we can write the corresponding channel matrix as

$$
\mathbf{H}^{m}=\left(\begin{array}{cccc}
h_{1,1}^{m} & h_{1,2}^{m} & \ldots & h_{1, T x}^{m} \\
h_{2,1}^{m} & h_{2,2}^{m} & \ldots & h_{2, T x}^{m} \\
\cdot & \cdot & \ldots & \cdot \\
\cdot & \cdot & \ldots & \cdot \\
\cdot & \cdot & \ldots & \cdot \\
h_{R x, 1}^{m} & h_{R x, 2}^{m} & \ldots & h_{R x, T x}^{m}
\end{array}\right)
$$

where $h_{R x, T x}^{m}$ is the CIR of the transmission link from transmitter $T x$ to receiver $R x$ at a channel delay of $m$. Consequently, we can write the channel matrix of the MIMO wideband channel as

$$
\mathbf{H}=\left(\mathbf{H}^{0}, \mathbf{H}^{1}, \ldots, \mathbf{H}^{N_{c}}\right)
$$

where $N_{c}$ is the maximum delay of the wideband channel.

The MMSE MIMO DFE feedforward filter matrix is given by solving the following system of equations for $\mathbf{S}$ [43]:

$$
\left(\mathbf{F F}^{H}+\Psi\right) \cdot\left(\begin{array}{c}
\mathbf{S}_{0}^{H} \\
\mathbf{S}_{1}^{H} \\
\cdot \\
\cdot \\
\cdot \\
\mathbf{S}_{N_{f}}^{H}
\end{array}\right)=\left(\begin{array}{c}
\mathbf{H}_{N_{f}} \\
\mathbf{H}_{N_{f}-1} \\
\cdot \\
\cdot \\
\cdot \\
\mathbf{H}_{0}
\end{array}\right)
$$

where $\mathbf{S}^{H}$ denotes the Hermitian transpose of $\mathbf{S}$ and $N_{f}$ is the forward filter length, $\mathbf{F}$ is defined by

$$
\mathbf{F}=\left(\begin{array}{ccccccc}
\mathbf{H}^{0} & \mathbf{H}^{1} & \mathbf{H}^{2} & . & . & . & \mathbf{H}^{N_{f}-1} \\
\mathbf{0} & \mathbf{H}^{0} & \mathbf{H}^{1} & . & . & . & \mathbf{H}^{N_{f}-2} \\
\mathbf{0} & \mathbf{0} & \mathbf{H}^{0} & . & . & . & \mathbf{H}^{N_{f}-3} \\
. & . & . & . & & & . \\
. & . & . & & . & & . \\
. & . & . & & . & . \\
\mathbf{0} & \mathbf{0} & \mathbf{0} & . & . & . & \mathbf{0}
\end{array}\right)
$$

TABLE I

PARAmeters of the SPACE-Time Trellis Codes Proposed IN [30]

\begin{tabular}{c|c|c|c|c|c}
\hline $\begin{array}{c}\text { Modulation } \\
\text { scheme }\end{array}$ & BPS & $\begin{array}{c}\text { Decoding } \\
\text { algorithm }\end{array}$ & $\begin{array}{c}\text { No. of } \\
\text { states }\end{array}$ & $\begin{array}{c}\text { No. of } \\
\text { transmitters }\end{array}$ & $\begin{array}{c}\text { No. of } \\
\text { termination } \\
\text { symbols }\end{array}$ \\
\hline 4PSK & 2 & VA & 16 & 2 & 2 \\
\hline 8PSK & 3 & VA & 16 & 2 & 2 \\
\hline 16PSK & 4 & VA & 16 & 2 & 1 \\
\hline
\end{tabular}

and $\Psi$ is defined by

$$
\Psi=\left(\begin{array}{cccccc}
N_{0} & 0 & . & . & . & 0 \\
0 & N_{0} & . & & & 0 \\
. & . & . & & & . \\
. & . & & . & & . \\
. & . & & & . & . \\
0 & 0 & . & . & . & N_{0}
\end{array}\right)
$$

where $N_{0}$ is the noise spectral density.

After the feedforward coefficients have been calculated using (4), we can find the $N_{b}$ number of backward coefficients with the aid of [43]

$$
Q_{n}=\sum_{m=\max \left(0, n-N_{c}+N_{f}-1\right)}^{\min \left(N_{f}, N_{f}+n+1\right)} \mathbf{S}_{m} \cdot \mathbf{H}_{N_{f}+1-m+n}
$$

\section{Simulation PARAMETERS}

The space-time trellis codes employed in this treatise were proposed by Tarokh et al. in [30]. We chose 4PSK, 8PSK, and 16PSK space-time trellis codes, since they provide the throughput of 2, 3, and 4 BPS, respectively. The associated parameters of the space-time trellis employed are summarized in Table I.

As we can see in Fig. 1, turbo codes, which were introduced by Berrou et al. [46], are concatenated with the AQAM scheme. Both convolutional and block codes can be used as component codes in a turbo code. It was shown by Hagenauer et al. [47] that turbo block codes outperform punctured turbo convolutional codes, when the coding rate is higher than $R=2 / 3$. It was also observed that a rate $R=0.98$ a turbo Hamming code using BPSK modulation is capable of operating within $0.27 \mathrm{~dB}$ of the Shannon limit [48] when communicating over the nondispersive Gaussian channel. In this contribution, we will investigate the performance of turbo

$$
\begin{aligned}
\gamma_{\mathrm{dfe}} & =\frac{\text { Wanted Signal Power }}{\text { Residual ISI Power }+ \text { Effective Noise Power }} \\
& =\frac{E\left[\left|S_{k} \sum_{m=0}^{N_{f}} C_{m} h_{m}\right|^{2}\right]}{S_{k-q} \sum_{q=-\left(N_{f}-1\right)}^{-1} E\left[\left|\sum_{m=0}^{N_{f}-1} C_{m} h_{m+q}\right|^{2}\right]+N_{o} \sum_{m=0}^{N_{f}}\left|C_{m}\right|^{2}}
\end{aligned}
$$


TABLE II

PARAMETERS OF THE DIFFERENT TURBO CODES USED IN FIG. 1

\begin{tabular}{l|c|c|c|c}
\hline Code & $\begin{array}{c}\text { Octal } \\
\text { generator } \\
\text { polynomial }\end{array}$ & $\begin{array}{c}\text { No. } \\
\text { of } \\
\text { states }\end{array}$ & $\begin{array}{c}\text { Decoding } \\
\text { algorithm }\end{array}$ & $\begin{array}{c}\text { No. } \\
\text { of }\end{array}$ \\
\hline \multicolumn{5}{c}{ iterations } \\
\hline TCurbo Convolutional Code (TC) \\
\hline \multicolumn{5}{c|}{ Turbo BCH Code (TBCH) } \\
\hline $\operatorname{TBCH}(31,26)$ & 45 & 32 & Log-Map & 8 \\
\hline $\operatorname{TBCH}(63,57)$ & 103 & 64 & Log-Map & 8 \\
\hline $\operatorname{TBCH}(127,120)$ & 211 & 128 & Log-Map & 8 \\
\hline
\end{tabular}

BCH (TBCH) [29] and turbo convolutional (TC) codes [29] when using various fixed modulation schemes and AQAM. Table II shows the parameters of each turbo codec used in the system.

As we can see in Table II, different BCH component codes are employed and this results in different coding rates. On the other hand, puncturing is applied to the TC code, which also results in different coding rates. We show in Table III the puncturing patterns employed and the result coding rates. The puncturing patterns seen in the table consist of two parts. Specifically, the associated different puncturing patterns represent the puncturing patterns of the parity bits emanating from the first and second decoder, respectively. Note that the puncturing patterns employed in these systems have been determined experimentally, but they are not claimed to be optimal. For procedures on designing high-rate turbo codes with the aid of puncturing, the interested reader is referred to [49]. Different modulation schemes were employed, which consequently result in different effective BPS throughputs, as shown in Table III. Prior research indicates that the bits mapped to the nonbinary symbols representing the phasors of a QAM constellation exhibit different BERs [37]. These different BER subchannels are also often referred to as the different protection classes of the QAM constellation. When combining QAM with channel coding, both the parity and the original information bits can be mapped to the different protection classes. In our recent work in [29], [50], and [51], we found that the corresponding best mapping rule also depends on the error correcting power, i.e., the contraint length of the convolutional constituent codes of the turbo codec used. Hence, for the sake of simplicity in this contribution, we randomly mapped the data and parity bits to the QAM constellation points.

The random turbo interleaver and random channel interleaver depths are limited such that each AQAM transmission burst can be individually decoded, i.e., burst-by-burst (BbB) turbo decoding is used. This may be important in low-delay interactive speech [52] and video systems [53]. Additionally, in an effort to quantify the best possible performance of the system, we also study a long-delay system, where the channel interleaver depth is chosen to be approximately $10^{5}$ bits in the context of all AQAM modes. The associated channel interleaver depth and the
TABLE III

Simulation Parameters AsSOCIATED With THE TC CHANNEL CODEC IN FIG. 1

\begin{tabular}{|c|c|c|c|c|c|c|}
\hline Code & $\begin{array}{l}\text { Code } \\
\text { Rate } \\
\quad R\end{array}$ & $\begin{array}{c}\text { Puncturing } \\
\text { Pattern }\end{array}$ & $\begin{array}{l}\text { Modula- } \\
\text { tion } \\
\text { Mode }\end{array}$ & BPS & $\begin{array}{l}\text { Random } \\
\text { turbo } \\
\text { interleaver } \\
\text { depth }\end{array}$ & $\begin{array}{c}\text { Random } \\
\text { channel } \\
\text { interleaver } \\
\text { depth }\end{array}$ \\
\hline \multirow[t]{22}{*}{$\mathrm{TC}(2,1,3)$} & \multirow[t]{3}{*}{0.33} & \multirow[t]{3}{*}{1,1} & BPSK & 0.33 & 228 & 684 \\
\hline & & & QPSK & 0.66 & 456 & 1368 \\
\hline & & & \multicolumn{2}{|c|}{ Long delay } & 34198 & 102600 \\
\hline & \multirow[t]{4}{*}{0.50} & \multirow[t]{4}{*}{10,01} & BPSK & 0.50 & 342 & 684 \\
\hline & & & QPSK & 1.00 & 684 & 1368 \\
\hline & & & 16QAM & 2.00 & 1368 & 2736 \\
\hline & & & \multicolumn{2}{|c|}{ Long delay } & 51300 & 102600 \\
\hline & \multirow[t]{5}{*}{0.75} & \multirow{5}{*}{$\begin{array}{l}100000001000 \\
001000000010\end{array}$} & BPSK & 0.75 & 513 & 684 \\
\hline & & & QPSK & 1.50 & 1026 & 1368 \\
\hline & & & 16QAM & 3.00 & 2052 & 2736 \\
\hline & & & 64QAM & 4.50 & 3078 & 4104 \\
\hline & & & \multicolumn{2}{|c|}{ Long delay } & 76950 & 102600 \\
\hline & \multirow[t]{5}{*}{0.83} & \multirow{5}{*}{$\begin{array}{l}1000000000 \\
0000000001\end{array}$} & BPSK & 0.83 & 570 & 684 \\
\hline & & & QPSK & 1.66 & 1140 & 1368 \\
\hline & & & 16QAM & 3.32 & 2280 & 2736 \\
\hline & & & 64QAM & 4.98 & 3420 & 4104 \\
\hline & & & \multicolumn{2}{|c|}{ Long delay } & 85500 & 102600 \\
\hline & \multirow[t]{5}{*}{0.90} & \multirow{5}{*}{$\begin{array}{l}000001000000000000 \\
000000000000100000, \\
100000000000000000 \\
000001000000000000\end{array} \mid$} & BPSK & 0.90 & 615 & 684 \\
\hline & & & QPSK & 1.80 & 1230 & 1368 \\
\hline & & & 16QAM & 3.60 & 2462 & 2736 \\
\hline & & & 64QAM & 5.40 & 3694 & 4104 \\
\hline & & & \multicolumn{2}{|c|}{ Long delay } & 92340 & 102600 \\
\hline
\end{tabular}

corresponding turbo interleaver depth is indicated by the "long delay" phrase in Table III.

Similarly to Table III, we show in Table IV the corresponding coding rates and the achievable BPS throughput of the TBCH codes employing various $\mathrm{BCH}$ component codes. Again, the turbo interleaver sizes were chosen for enabling BbB turbo decoding. Additionally, both the turbo and channel interleaver depths of the delay nonsensitive system are also shown in Table IV, again, indicated as the "long delay" scenario.

Our studies were conducted for transmission over the COST 207 typical urban (TU) [54] channel. The corresponding CIR is shown in Fig. 2. Each path was faded independently according to the Rayleigh distribution and the corresponding normalized Doppler frequency was $3.27 \times 10^{-5}$. Both the SISO and the MIMO DFE incorporated 35 forward taps and seven feedback taps and the transmission burst structure used in our treatise is shown in Fig. 3. The following assumptions were stipulated. Firstly, the CIR was time invariant for the duration of a transmission burst but varied from burst to burst, which corresponds to assuming that the CIR is slowly varying. Secondly, perfect channel estimation and perfect knowledge of the AQAM mode used was assumed at the receiver. In practice, a simple repetition code can be used for conveying the AQAM 
TABLE IV

Simulation PaRAMETERS ASSOCIATED With the TBCH CHANNEL CODEC IN FIG. 1

\begin{tabular}{|c|c|c|c|c|c|c|}
\hline Code & $\begin{array}{c}\text { Code } \\
\text { Rate } \\
R\end{array}$ & $\begin{array}{c}\text { Puncturing } \\
\text { Pattern }\end{array}$ & $\begin{array}{c}\text { Modula- } \\
\text { tion } \\
\text { Mode }\end{array}$ & BPS & $\begin{array}{c}\text { Random } \\
\text { turbo } \\
\text { interleaver } \\
\text { depth }\end{array}$ & $\begin{array}{c}\text { Random } \\
\text { channel } \\
\text { interleaver } \\
\text { depth }\end{array}$ \\
\hline \multirow[t]{5}{*}{ TBCH $(31,26)$} & \multirow[t]{5}{*}{0.72} & \multirow[t]{5}{*}{1,1} & BPSK & 0.72 & 494 & 684 \\
\hline & & & QPSK & 1.44 & 988 & 1368 \\
\hline & & & 16QAM & 2.88 & 1976 & 2736 \\
\hline & & & 64QAM & 4.32 & 2964 & 4104 \\
\hline & & & \multicolumn{2}{|c|}{ Long delay } & 74100 & 102600 \\
\hline \multirow[t]{5}{*}{$\operatorname{TBCH}(63,57)$} & \multirow[t]{5}{*}{0.83} & \multirow[t]{5}{*}{1,1} & BPSK & 0.83 & 513 & 621 \\
\hline & & & QPSK & 1.66 & 1083 & 1311 \\
\hline & & & 16QAM & 3.32 & 2223 & 2691 \\
\hline & & & 64QAM & 4.98 & 3363 & 4071 \\
\hline & & & \multicolumn{2}{|c|}{ Long delay } & 77976 & 94392 \\
\hline \multirow[t]{5}{*}{$\mathrm{TBCH}(127,120)$} & \multirow[t]{5}{*}{0.90} & \multirow[t]{5}{*}{1,1} & BPSK & 0.90 & 600 & 670 \\
\hline & & & QPSK & 1.80 & 1200 & 1340 \\
\hline & & & 16QAM & 3.60 & 2400 & 2680 \\
\hline & & & 64QAM & 5.40 & 3600 & 4020 \\
\hline & & & Long $d$ & elay & 82080 & 91656 \\
\hline
\end{tabular}

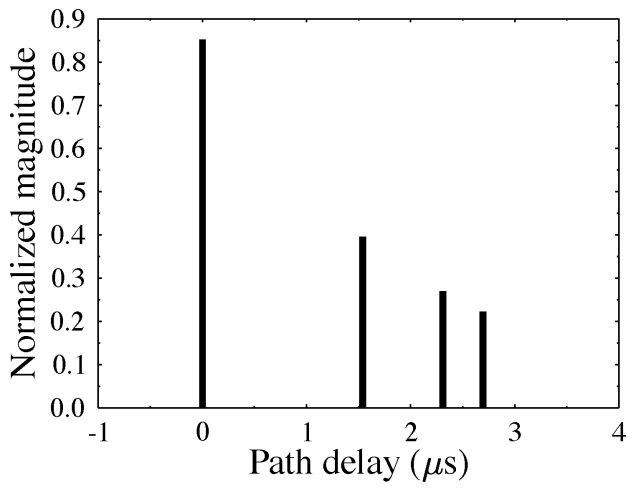

Fig. 2. Impulse response of a COST 207 TU channel [54].

mode to the receiver. ${ }^{1}$ Furthermore, the modulation mode can also be detected blindly [13], [14]. Thirdly, the pseudo-SNR at the output of the equalizer is estimated perfectly prior to transmission, which, again, tacitly assumes the existence of a reliable low-delay feedback path between the transmitter and the receiver. In practice, there will be error propagation in the DFE's feedback loop, which will degrade the performance of the system. ${ }^{2}$

${ }^{1}$ For example, three bits are requited for signaling one of the five possible AQAM modes, which may be repeated three times for the sake of adding protection. The receiver's majority logic decision (MLD) scheme is capable of recovering the correct AQAM mode, even if three out of nine bits are erroneous, which corresponds to a channel BER of 33\%. Alternatively, Torrance's unequal protection scheme [55] can be used, which requires less redundancy.

${ }^{2}$ We note, however, that the AQAM switching thresholds can be appropriately increased, in order to compensate for the error propagation phenomenon of the DFE at the cost of a slight reduction of the system's BPS throughput.

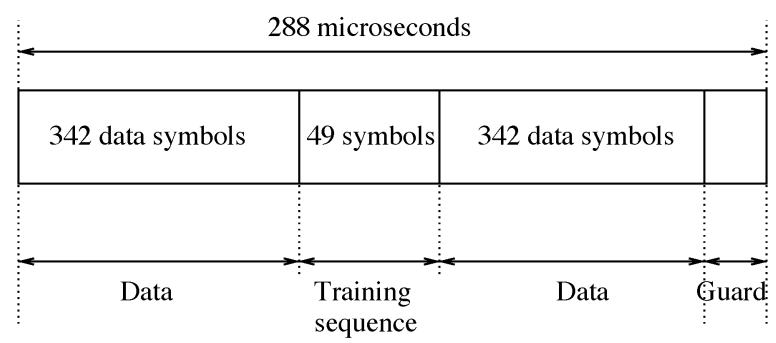

non-spread data burst

Fig. 3. Transmission burst structure of the FMA1 nonspread data.

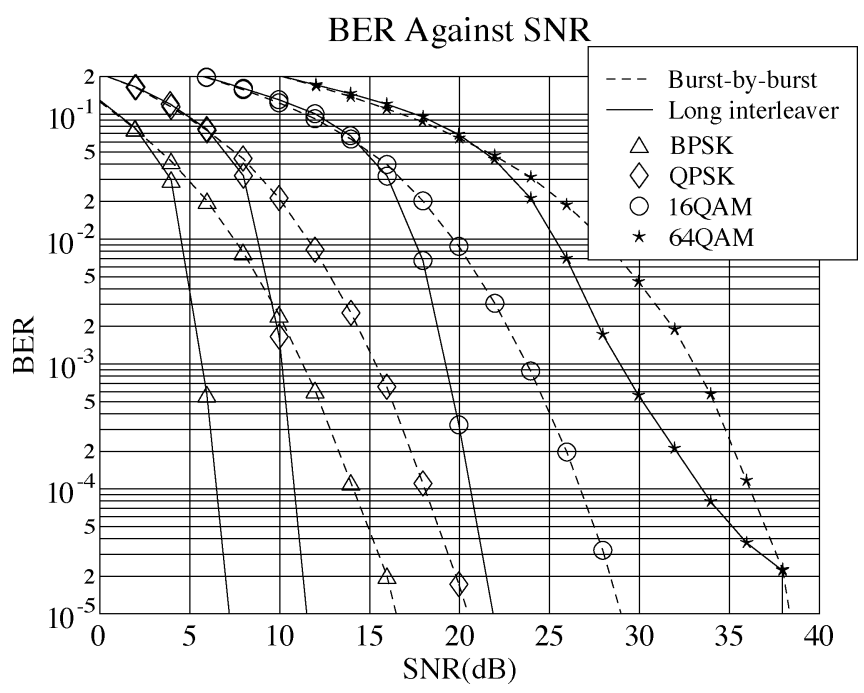

Fig. 4. BER performance comparison between rate $R=0.75 \mathrm{TC} \mathrm{BbB}$ decoding and TC coding using the long channel interleaver for each individual modulation mode, when communicating over the Rayleigh fading COST207 TU channel of Fig. 2. Associated coding parameters are shown in Tables II and III

\section{Simulation Results}

\section{A. Turbo-Coded Fixed Modulation Mode Performance}

Before analyzing the proposed AQAM schemes, we shall investigate the BER performance of the turbo-coded system utilizing the fixed modulation modes of BPSK, QPSK, 16QAM, and 64QAM. The effect of the channel interleaver length, turbo component codes, coding rate, and DFE error propagation on the BER performance will be investigated.

Fig. 4 shows the BER performance of the rate $R=0.75$ TC coded fixed modulation schemes for both the short channel interleaver, which enables $\mathrm{BbB}$ decoding, and for the long channel interleaver, which has a delay of $10^{5}$ bits. $^{3}$ As expected, in Fig. 4 the BER performance associated with using the longer channel interleaver was superior compared to that using the shorter channel interleaver, although naturally at the cost of an associated higher transmission delay. Except for 64QAM, there was more than 5-dB "interleaver-gain" at a BER of $10^{-4}$, when using the longer channel interleaver. The poor performance of the system, when 64QAM was used, was due to the error propagation of the DFE, which significantly

${ }^{3}$ The rationale of this experiment is to resolve, whether the agile $\mathrm{BbB}$ reconfiguration of the AQAM modes or the employment of a long burst-randomizing interleaver is more important. 


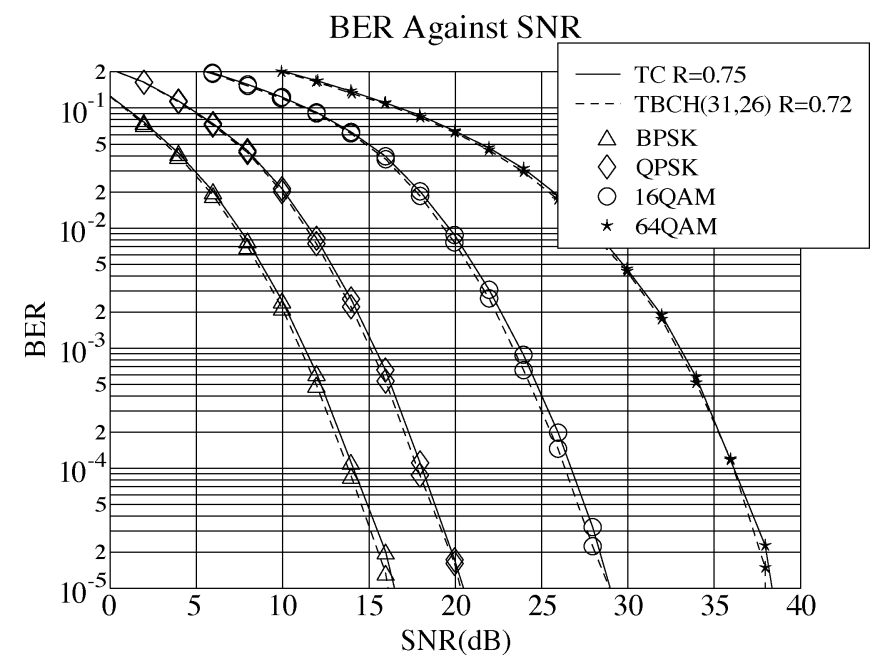

Fig. 5. BER performance comparison between the rate $R=0.75 \mathrm{TC}$ and the $R=0.72 \mathrm{TBCH}$ codes using $\mathrm{BbB}$ decoding for each individual modulation mode for transmission over the Rayleigh fading COST207 TU channel of Fig. 2. Associated coding parameters are shown in Tables II- IV.

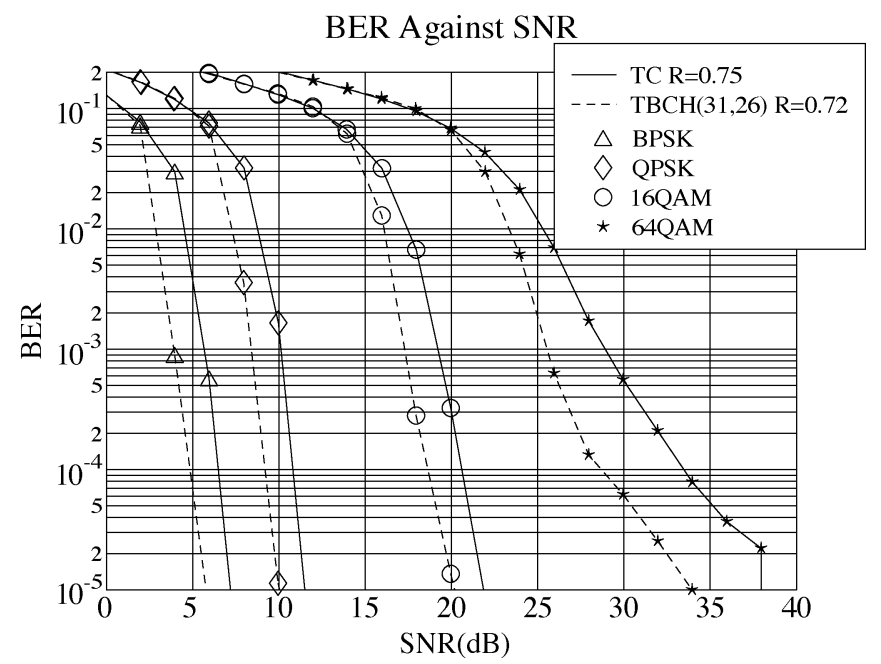

Fig. 6. BER performance comparison between the rate $R=0.75 \mathrm{TC}$ and the rate $R=0.72 \mathrm{TBCH}$ codes using the $10^{5}$ bit delay channel interleaver for each individual modulation mode for transmission over the Rayleigh fading COST207 TU channel of Fig. 2. Associated coding parameters are shown in Tables II-IV.

degraded the performance of the system and hence overloaded error correction capability of the turbo codes.

In Fig. 5, we compare the performance of $\mathrm{TC}$ and $\mathrm{TBCH}$ codes having similar coding rates. The channel interleaver length was chosen such that it enables $\mathrm{BbB}$ decoding at the receiver. As we can see in Fig. 5, there is virtually no performance difference between the $\mathrm{TC}$ and $\mathrm{TBCH}$ codes in conjunction with the various modulation schemes studied.

Similarly to Fig. 5, in Fig. 6 we compare the performance of TC and TBCH codes, although in Fig. 6 the channel interleaver's length was chosen to be approximately $10^{5}$ bits. Unlike in Fig. 5, we can clearly see that the performance of the rate $R=0.72 \mathrm{TBCH}$ code becomes better than that of the TC code. At a BER of $10^{-4}$ we can observe from Fig. 6 that there is an SNR gain of about $2 \mathrm{~dB}$ for the TBCH code compared to the TC code. From Figs. 5 and 6, we can conclude that the rate

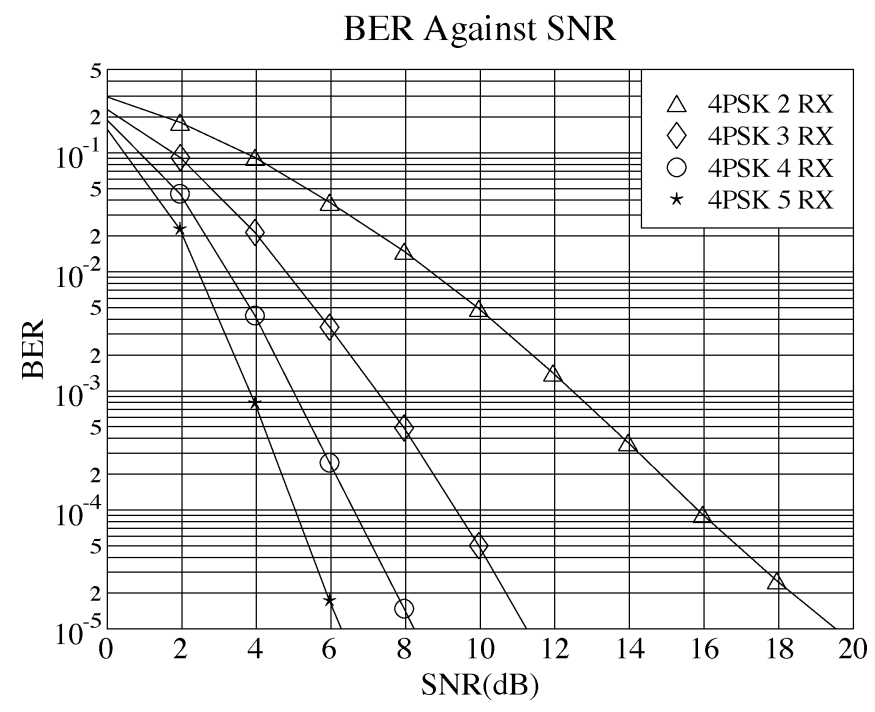

Fig. 7. BER performance comparison between various 4PSK space-time trellis codes using 2, 3, 4, and 5 receivers for transmission over the Rayleigh fading COST207 TU channel of Fig. 2. Associated coding parameters are shown in Table I.

$R=0.72 \mathrm{TBCH}$ code outperforms the rate $R=0.75 \mathrm{TC}$ code, when a channel interleaver length of $10^{5}$ bits is employed in the system.

\section{B. Space-Time Trellis Code Performance}

In the previous section, we presented our simulation results for various turbo-coded systems utilizing fixed modulation modes. Let us now extend our investigations into the behavior of space-time trellis codes. As argued in Section II, since multiple transmitters and receivers are employed, a MIMO equalizer will be employed for equalizing the MIMO channels. Since two transmitters are employed for the space-time trellis code used, there has to be a minimum of two receivers employed [43].

In Fig. 7, we show the BER performance of a range of 4PSK space-time trellis codes using 2 to 5 receivers [30]. At a BER of $10^{-4}$, we observe that there is a huge SNR improvement of more than $15 \mathrm{~dB}$, when the number of receivers is increased from two to three. As the number of receivers is further increased to four and five, the incremental performance gain becomes smaller and smaller. Similar trends can be observed also for 8 and 16PSK space-time trellis codes.

\section{AQAM Performance}

In the previous sections, we have shown simulation results for turbo-coded systems communicating using fixed modulation modes. Furthermore, we also presented performance results for the space-time trellis codes employed in conjunction with a MIMO equalizer. Let us now study the required SNR of the turbo-coded fixed modem mode-based system and that of the space-time trellis codes comparatively in terms of their achievable BPS throughput, when aiming for a target BER of $10^{-4}$. These results will then be compared to the BPS performance of the proposed AQAM schemes at a BER of $10^{-4}$ in Fig. 8.

Explicitly, there are two sets of curves in Fig. 8. The curves drawn in a continuous line represent the system having no 


\section{BPS Against SNR}

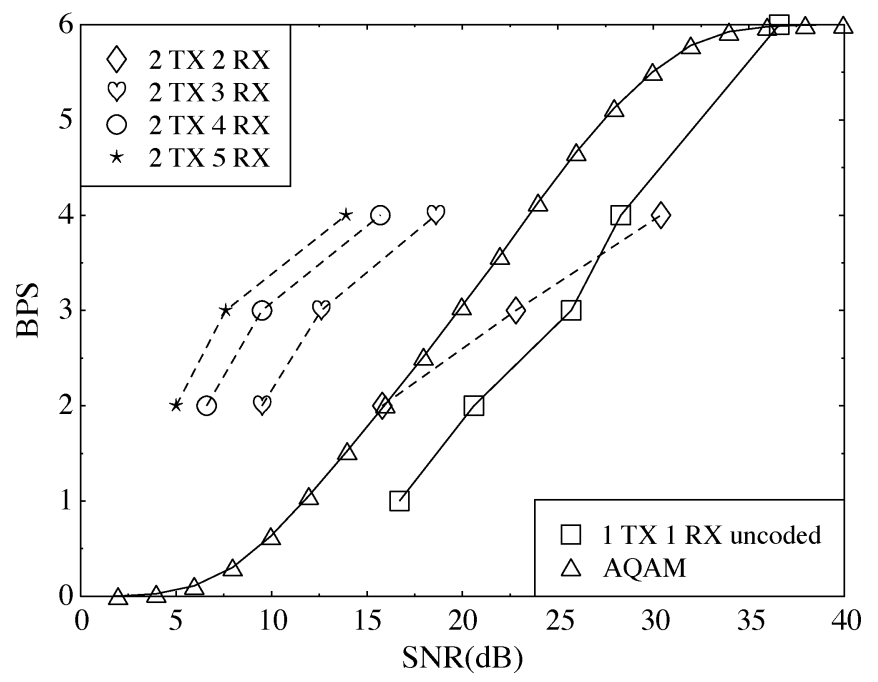

Fig. 8. BPS performance comparison between space-time trellis codes, uncoded AQAM, and the uncoded fixed modulation modes when communicating over the Rayleigh fading COST207 TU channel of Fig. 2. Associated coding parameters are shown in Table I and the target BER is $10^{-4}$.

diversity gain, i.e., that employing one transmitter and one receiver. By contrast, the curves drawn using a broken line represent the system having diversity gain due to employing space-time trellis codes and multiple receivers. The curve marked with squares represents the performance of the uncoded system using 1, 2, 3, 4, and 6 BPS fixed modulation schemes. In [10], an uncoded wideband BbB adaptive modem was characterized and its performance was reproduced in Fig. 8, which is denoted by the triangles. We can see in Fig. 8 that by employing space-time trellis codes in conjunction with two receivers, we have an SNR gain of $5 \mathrm{~dB}$ at a throughput of 2 BPS as compared to the uncoded system. The achievable SNR performance gain reduces to $3 \mathrm{~dB}$, when the required throughput of the system is increased to $3 \mathrm{BPS}$, and there is a negative SNR gain when the required system throughput is 4 BPS. When employing AQAM, the single-transmitter and single-receiver system outperforms the space-time trellis code using two receivers. By contrast, the AQAM BPS performance remains inferior to that of the space-time trellis code using three receivers, as shown in Fig. 8.

In Fig. 8, we have shown the performance of the uncoded onetransmitter and one-receiver fixed modulation scheme, which was outperformed by the BbB AQAM scheme proposed in [10]. We will now investigate the achievable BPS versus SNR gain due to employing turbo codes. In Fig. 9, we show the BPS performance of both TC and TBCH codes for the short and long channel interleaver scenarios. ${ }^{4}$ Recall that the length of the short channel interleaver was chosen such that it enabled $\mathrm{BbB}$ turbo decoding, while the long channel interleaver depth was approximately $10^{5}$ bits. We have shown in Fig. 5 that there is no significant performance difference between the TC and TBCH codes for BbB turbo decoding, which is also reflected in Fig. 9. More explicitly, it is shown in Fig. 9 that both BbB TC

\footnotetext{
${ }^{4}$ The associated curve was generated by combining all the different throughput fixed mode modulation schemes with all the different coding rates schemes.
}

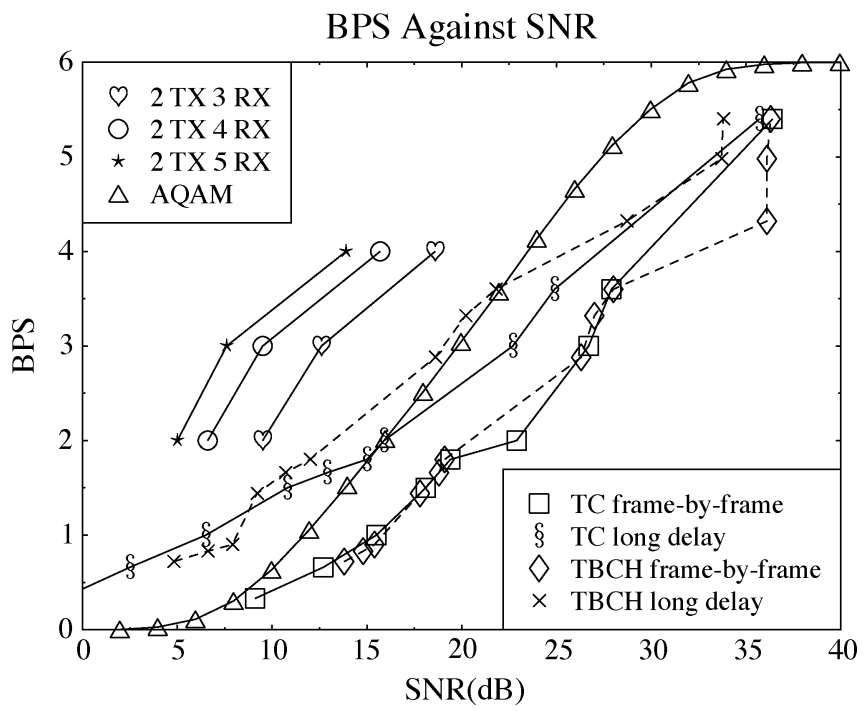

Fig. 9. BPS performance comparison between space-time trellis codes, uncoded AQAM, TC, and TBCH codes in conjunction with both BbB decoding and long delay channel interleaving for transmission over the Rayleigh fading COST207 TU channel of Fig. 2. Associated coding parameters are shown in Tables I-IV and the target BER is $10^{-4}$.

and TBCH decoding exhibit a similar BPS performance, as evidenced by the overlapping curves marked with diamonds and squares, respectively. It can be seen that BbB turbo decoding in conjunction with fixed modulation is inferior to the uncoded adaptive modulation scheme. If, however, the delay permitted by the system allows the employment of a channel interleaver length of approximately $10^{5}$ bits, we can clearly see in Fig. 9 that there is a substantial SNR improvement over the BbB scenario using the same modulation schemes. For TC codes, we can see that the system outperforms uncoded AQAM for throughput values below 2 BPS. At 0.5 BPS, we can see in Fig. 9 that the TC code outperforms the uncoded AQAM scheme by approximately $10 \mathrm{~dB}$. On the other hand, if TBCH codes are employed we can see that the system outperforms the uncoded AQAM scheme up to throughput values as high as 3.5 BPS.

In Fig. 9, we have investigated the performances of TC and TBCH codes in conjunction with fixed modulation-based systems for both low- and high-channel interleaver lengths. Let us now fully exploit the error correction capability of turbo codes in the context of AQAM. In our earlier research, we used a set of fixed AQAM thresholds, which was determined with the aid of Powell's optimization [56]. However, the achievable throughput of the system can be further increased when we optimize the AQAM switching thresholds for each individual channel SNR value using Lagrangian optimization [17]. We note, however, that the solutions in [17] use no channel coding during the optimization of the AQAM thresholds. Since at the time of writing no analytical formula exists for the BER of turbo codes, employing Lagrangian optimization might not be feasible for AQAM systems employing turbo codes. Hence, in our proposed turbo-coded system we propose a simple procedure for determining the switching thresholds for each average channel SNR value.

In Fig. 10, we show the flow chart of the simple procedure proposed for determining the AQAM switching thresholds. As we can see in the figure, we commence the procedure by setting 


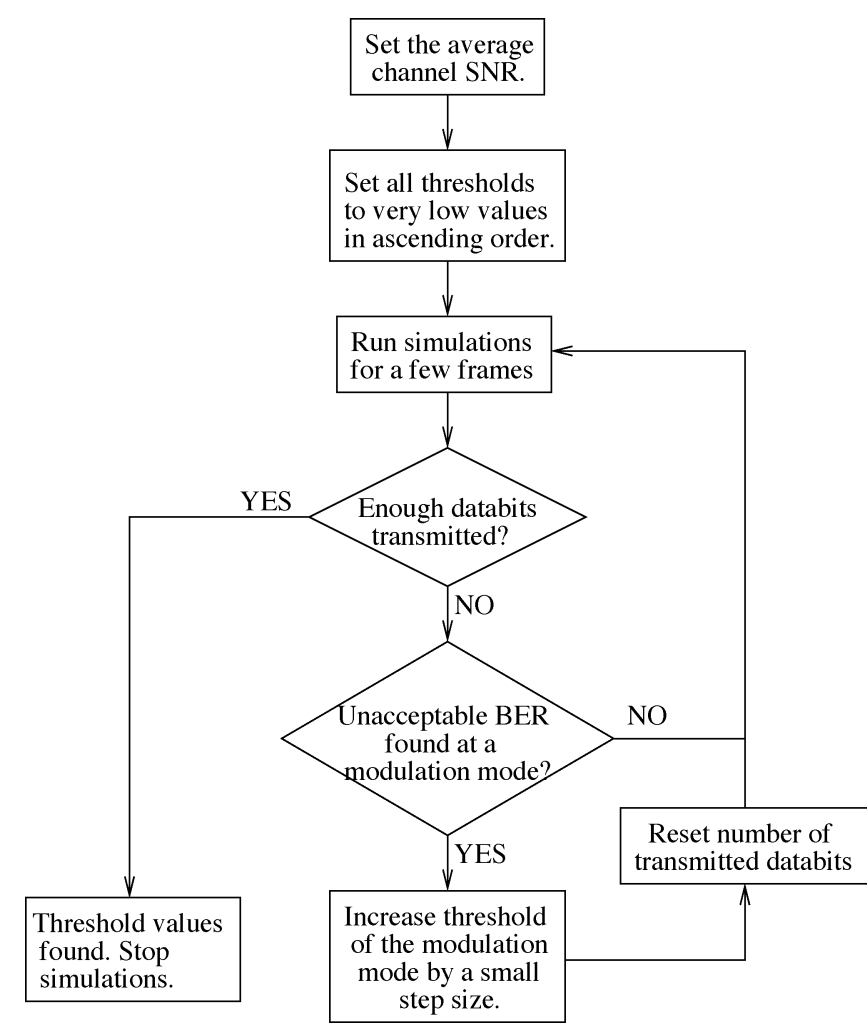

Fig. 10. Procedure for determining the AQAM switching thresholds.

the channel SNR, where the switching thresholds will be determined. Then, we assign the lowest possible SNR switching threshold to each AQAM mode in an ascending order. At this stage, the switching thresholds are set so low that the simulated BER will be significantly higher than the targeted BER. When the simulations are started and a few frames have been received, we check whether sufficient data bits were transmitted, such that a statistically relevant BER result can be obtained. If not, the BER of each AQAM mode is checked, commencing with the lowest throughput modulation mode. Once the statistically relevant BER was found in a particular AQAM mode, the switching threshold of the modulation mode will be incremented by a small step size. Then, the number of transmitted data bits is reset to zero and the simulations are restarted. On the other hand, if there is no modulation mode having a sufficiently reliable BER estimate, more data bits will be transmitted. The entire process is repeated until a sufficiently high number of data bits has been transmitted for generating a statistically pertinent average BER result.

In Fig. 11, we show the BER and BPS performance of $\mathrm{BbB}$ TC decoding using various coding rates and adaptive modulation. The above-mentioned simple procedure was used for determining the switching thresholds. As we can see in Fig. 11, the coded BER of the AQAM modem using various TC coding rates was maintained at an average of $10^{-4}$. If the same fixed SNR-independent switching thresholds were employed for every channel SNR, we would have an undulating coded BER curve, which would clearly deviate from the targeted constant BER. In Fig. 11, we also plotted the BPS performance associated with each TC coding rate, which is scaled on the right-hand side axis. As we can see in Fig. 11, at

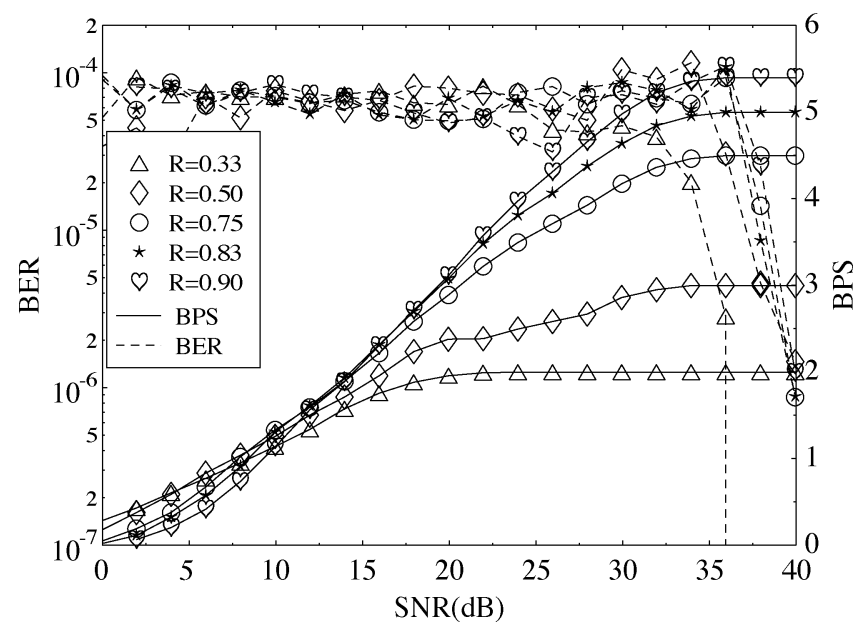

Fig. 11. BER and BPS performance of BbB TC decoding using various coding rates and adaptive modulation, when communicating over the Rayleigh fading COST207 TU channel of Fig. 2. Associated coding parameters are shown in Tables II and III and the target BER is $10^{-4}$.

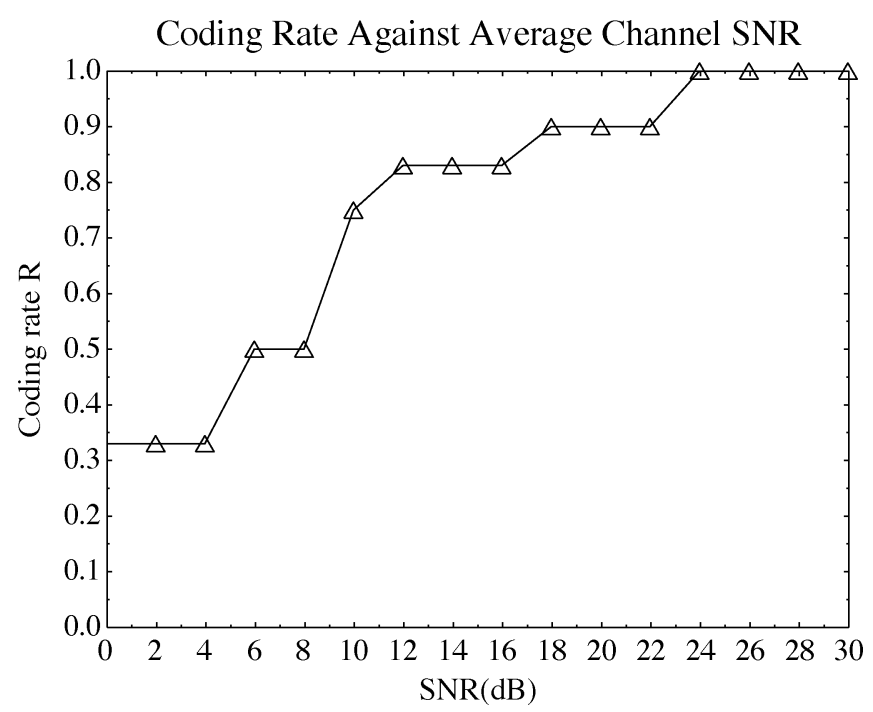

Fig. 12. TC coding rates activated in conjunction with AQAM for different channel SNRs when communicating over the Rayleigh fading COST207 TU channel of Fig. 2. Associated coding parameters are shown in Tables II and III and the target BER is $10^{-4}$.

low channel SNRs, the schemes having lower TC coding rates will outperform those associated with the higher TC coding rates. For example, TC codes having a rate of $R \leq 0.5$ have a BPS throughput in excess of 0.5 for channel SNRs above $4 \mathrm{~dB}$. However, as we increase the channel SNR, the TC-coded AQAM schemes having higher coding rates outperform their lower coding rate counterparts. Furthermore, the throughput of the AQAM schemes having lower TC coding rates will saturate faster and at a lower value. For example, the TC code associated with $R=1 / 3$ has a maximum BPS throughput of $6 \times 1 / 3=2$. From Fig. 11, we can conclude that when the channel SNR is low, lower TC coding rates have to be employed and $R$ should be increased as the channel SNR increases.

Based on Fig. 11, in our adaptive regime we select the specific TC coding rate for every channel SNR, which gives the maximum throughput in the context of the TC-coded AQAM system. In Fig. 12, we portray the TC coding rates selected at the various channel SNRs encountered. As we can see in the 


\section{BPS Against SNR}

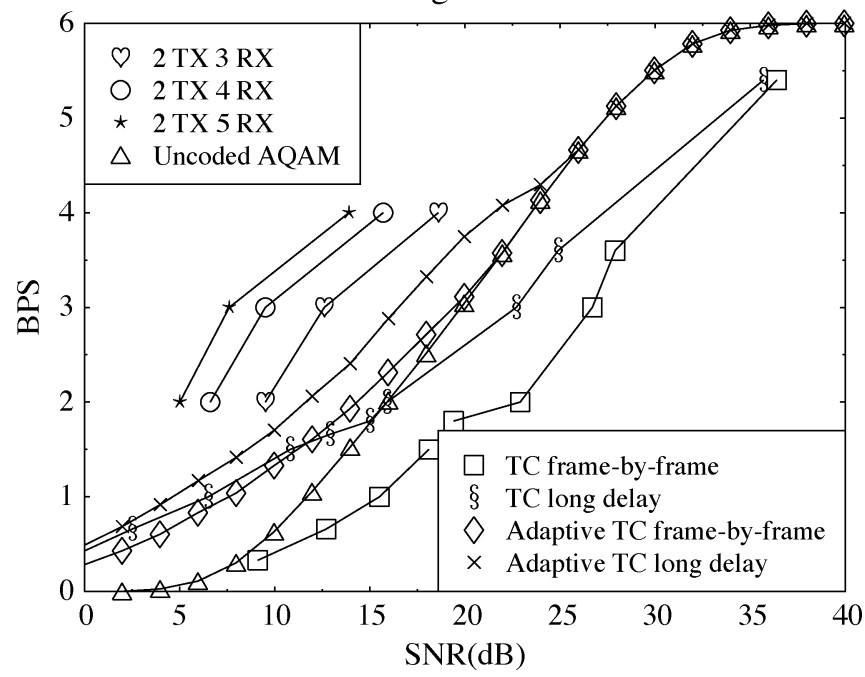

Fig. 13. BPS performance comparison between space-time trellis codes, uncoded AQAM, adaptive-rate TC codes using BbB decoding and long delay channel interleaver both with fixed and adaptive modulation, when communicating over the Rayleigh fading COST207 TU channel of Fig. 2. Associated coding parameters are shown in Tables I-III and the target BER is $10^{-4}$.

figure, the selected TC coding rate increases, as the channel SNR increases. For a channel SNR in excess of $24 \mathrm{~dB}$, we can see that the TC coding rate was set to unity, which implies that the BPS throughput of the uncoded AQAM scheme becomes better than that of the TC-coded AQAM scheme. Hence, TC coding is deactivated for channel SNRs in excess of 24 dB. Previously in Fig. 10, we proposed to optimize the AQAM switching thresholds individually for every channel SNR for turbo-coded AQAM. Let us now extend our proposal for optimizing not only the thresholds but also the TC coding rates for every channel SNR.

Let us hence apply the joint switching threshold and TC coding rate optimization techniques to the TC-coded AQAM system, which we refer to in our future discourse as optimum rate TC (ORTC)-aided AQAM. In Fig. 13, the curve marked with diamonds represents the BPS performance of the jointly optimized ORTC AQAM in conjunction with BbB decoding. As we can see in the figure, the employment of ORTC AQAM substantially improves the performance of the system, when the channel SNR is low. For example, at 0.5 BPS we observe a 5-dB SNR gain over the uncoded AQAM system. However, as the channel SNR is increased, the SNR gain due to TC coding is reduced and uncoded AQAM will be employed for channel SNRs in excess of $20 \mathrm{~dB}$. For channel SNRs below $13 \mathrm{~dB}$ we can observe in Fig. 13 that the performance of the ORTC AQAM scheme is similar to that of the TC coded fixed modulation schemes using the long-delay $10^{5}$-bit channel interleaver. Hence, with the aid of ORTC AQAM we can achieve as good a performance as the long-delay optimized-rate TC coded fixed modulation scheme, with the added benefit of incurring only a short delay. More explicitly, with the aid of the AQAM system we were able to reduce the interleaving delay without compromising the BPS throughput.

On the other hand, if a long delay is tolerable in the context of the ORTC AQAM system, the $10^{5}$-bit channel interleaver can be

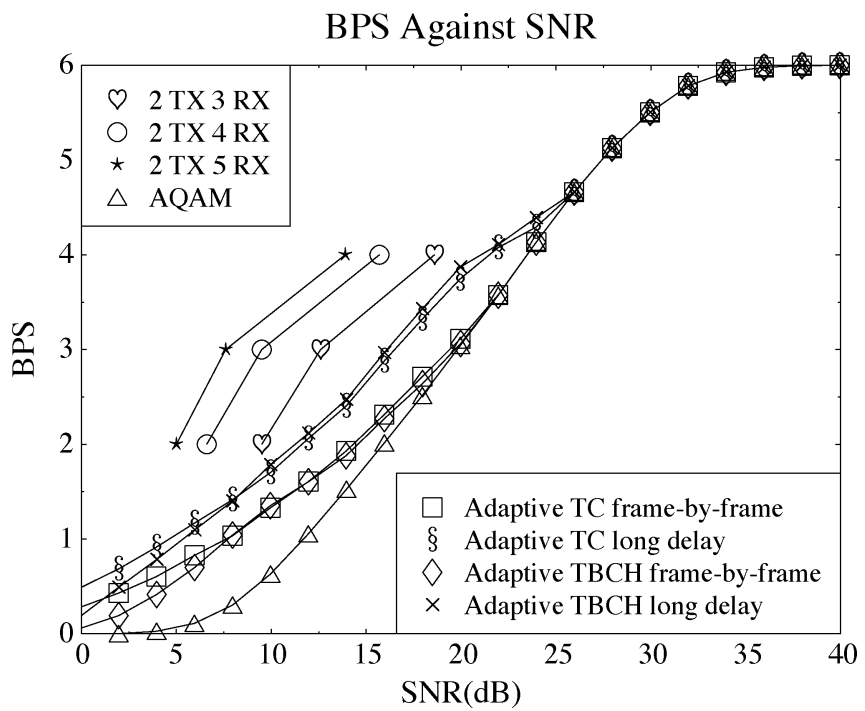

Fig. 14. BPS performance comparison between space-time trellis codes, uncoded AQAM, adaptive-rate TC, and TBCH codes using both BbB decoding and a long-delay channel interleaver employing fixed and adaptive modulation, when communicating over the Rayleigh fading COST207 TU channel of Fig. 2. Associated coding parameters are shown in Tables I-IV and the target BER is $10^{-4}$.

employed in the system. The corresponding BPS performance is shown in Fig. 13 by the curve marked with crosses. As we can see in the figure, there is a significant BPS performance improvement over the short-delay BbB ORTC AQAM system. Observe also in Fig. 13 that uncoded AQAM will only be employed for channel SNRs in excess of $25 \mathrm{~dB}$. The corresponding performance also approaches that of the space-time trellis codes using three receivers. At a throughput of 2 BPS we can see that the long-delay AQAM SNR performance is about $2-3 \mathrm{~dB}$ inferior to that of the space-time trellis codes using three receivers.

In Figs. 11 and 13, we have characterized the BPS performance of the ORTC AQAM system. Let us now present the corresponding results for the optimum rate TBCH $(\mathrm{ORTBCH})$ AQAM system and compare them to the ORTC AQAM system. As we can see in Fig. 14, the performance of the ORTC and ORTBCH codes is similar for the short-delay BbB turbo-decoded AQAM scheme, which we have also observed in Fig. 5. However, for lower channel SNRs the performance of ORTBCH AQAM is inferior to that of ORTC AQAM, since in this SNR region lower coding rate TC codes were employed.

On the other hand, we also show in Fig. 14 the performance of ORTC and ORTBCH AQAM using large channel interleavers. Similarly to BbB decoding, the TC codes outperform the TBCH codes for low channel SNRs. However, as the channel SNR is increased, we can see that the TBCH codes have an edge over the TC codes for channel SNRs in excess of $7 \mathrm{~dB}$, although their performance gap is insignificant as compared to those observed in Fig. 6. Therefore, we conclude that the more complex TBCH codes do not significantly outperform TC codes in the context of the AQAM system.

\section{CONCLUSION}

In this contribution, we comparatively studied the performance of space-time trellis codes and AQAM schemes. When multiple transmitters and receivers are employed, a MIMO 
equalizer has to be used for equalizing the multiple transmitted signals. On the other hand, a SISO equalizer was employed for the AQAM scheme, which used a single transmitter and single receiver. We then proposed to concatenate powerful turbo codes with the AQAM system.

The simulation results were presented in Section IV. In Section IV-A, we investigated the performance of the system using fixed modulation schemes. The performance of TC and TBCH codes was investigated and compared using both short and long channel interleavers. The length of the short channel interleaver was chosen such that $\mathrm{BbB}$ turbo decoding was used. The performance of TC codes in conjunction with various coding rates was presented and we found that the performance of TC codes improved as the coding rate was reduced. Then, in Section IV-B, we characterized the performance of 4, 8, and 16PSK space-time trellis codes.

Finally, in Section IV-C, we compiled all the results and compared them to the performance of the proposed turbo-coded AQAM system. Initially, we showed that the uncoded AQAM system outperforms the space-time trellis codes using two receivers. We then demonstrated that uncoded AQAM also outperforms BbB TC coded fixed modulation. However, in conjunction with a large channel interleaver the TC coded fixed modulation scheme outperforms the uncoded AQAM scheme for channel SNRs below $15 \mathrm{~dB}$ at the cost of a high delay. A turbo-coded AQAM system was then proposed and we jointly optimized the AQAM switching thresholds and the coding rates for the turbo-coded AQAM system. The performance of the ORTC AQAM scheme using a short channel interleaver was found to be similar to that of the TC coded long-delay fixed modulation scheme. Finally, at the expense of a high channel interleaver delay, we were able to further improve the performance of the ORTC AQAM scheme. It was found that its performance was $2 \mathrm{~dB}$ inferior in comparison to the space-time trellis codes using three receivers. The performance of the ORTBCH AQAM scheme was then compared to that of the ORTC AQAM scheme. It was found that there was no significant improvement over the ORTC AQAM scheme, despite the extra complexity incurred by the system. In conclusion, ORTC AQAM can be viewed as a lower complexity measure of mitigating the channel quality fluctuations of wireless channels in comparison to multiple transmitter and receiver based space-time codes. If the complexity of the latter schemes is affordable, the performance advantages of AQAM erode.

\section{REFERENCES}

[1] J. F. Hayes, "Adaptive feedback communications," IEEE Trans. Commun. Technol., vol. 16, pp. 29-34, Feb. 1968.

[2] J. K. Cavers, "Variable rate transmission for Rayleigh fading channels," IEEE Trans. Commun. Technol., vol. COM-20, pp. 15-22, Feb. 1972.

[3] W. T. Webb and R. Steele, "Variable rate QAM for mobile radio," IEEE Trans. Commun., vol. 43, pp. 2223-2230, July 1995.

[4] S. Otsuki, S. Sampei, and N. Morinaga, "Square QAM adaptive modulation/TDMA/TDD systems using modulation level estimation with Walsh function," Electron. Lett., vol. 31, pp. 169-171, Feb. 1995.

[5] A. Goldsmith and P. Varaiya, "Capacity of fading channels with channel side information," IEEE Trans. Inform. Theory, vol. 43, pp. 1986-1992, Nov. 1997.

[6] W. Lee, "Spectrum efficiency in cellular," IEEE Trans. Veh. Technol., vol. 38, pp. 69-75, May 1989.
[7] A. J. Goldsmith and S. Chua, "Variable-rate variable-power MQAM for fading channels," IEEE Trans. Commun., vol. 45, pp. 1218-1230, Oct. 1997.

[8] J. Torrance and L. Hanzo, "Optimization of switching levels for adaptive modulation in a slow Rayleigh fading channel," Electron. Lett., vol. 32, pp. 1167-1169, June 20, 1996.

[9] W. H. Press, S. A. Teukolsky, W. T. Vetterling, and B. P. Flannery, Numerical Recipes in C. Cambridge, U.K.: Cambridge Univ. Press, 1992.

[10] C. Wong and L. Hanzo, "Upper-bound performance of a wideband burst-by-burst adaptive modem," IEEE Trans. Commun., vol. 48, pp. 367-369, Mar. 2000.

[11] E. Kuan, S. Ng, and L. Hanzo, "Joint-detection and interference cancellation based burst-by-burst adaptive cdma schemes," IEEE Trans. Veh. Technol., vol. 51, pp. 1479-1493, Nov. 2002.

[12] E. Kuan and L. Hanzo, "Burst-by-burst adaptive multiuser detection CDMA: A framework for existing and future wireless standards," Proc. IEEE, vol. 91, pp. 275-302, Feb. 2002.

[13] T. Keller and L. Hanzo, "Adaptive multicarrier modulation: A convenient framework for time-frequency processing in wireless communications," Proc. IEEE, vol. 88, pp. 611-642, May 2000.

[14] - "Adaptive modulation technique for duplex OFDM transmission," IEEE Trans. Veh. Technol., vol. 49, pp. 1893-1906, Sept. 2000.

[15] B. J. Choi, M. Münster, L. L. Yang, and L. Hanzo, "Performance of Rake receiver assisted adaptive-modulation based CDMA over frequency selective slow Rayleigh fading channel," Electron. Lett., vol. 37, pp. 247-249, Feb. 2001.

[16] G. S. G. Beveridge and R. S. Schechter, Optimization: Theory and Practice. New York: McGraw-Hill, 1970.

[17] B. J. Choi and L. Hanzo, "Performance analysis of RAKE-receiver assisted adaptive qam using antenna diversity over frequency selective Rayleigh channels," IEEE Trans. Veh. Technol., vol. 52, pp. 536-560, May 2003.

[18] L. Hanzo, C. H. Wong, and M. S. Yee, Adaptive Wireless Transceivers. New York: Wiley, 2002.

[19] S. Nanda, K. Balachandran, and S. Kumar, "Adaptation techniques in wireless packet data services," IEEE Commun. Mag., vol. 38, pp. 54-64, Jan. 2000.

[20] J. Blogh and L. Hanzo, $3 G$ Systems and Intelligent Networking. New York: Wiley, 2002.

[21] L. Hanzo, M. Münster, B. Choi, and T. Keller, OFDM and MC-CDMA for Broadband Multiuser Communications, WLAN's and Broadcasting: Wiley, 2003.

[22] V. Lau and S. Maric, "Variable rate adaptive modulation for DS-CDMA,” IEEE Trans. Commun., vol. 47, pp. 577-589, Apr. 1999.

[23] L. Hanzo, L.-L. Yang, E.-L. Kuan, and K. Yen, Single- and Multi-Carrier DS-CDMA. New York: Wiley, 2003.

[24] M. Yee and L. Hanzo, "A wideband radial basis function decision feedback equaliser assisted burst-by-burst adaptive modem," IEEE Trans. Commun., vol. 50, pp. 693-697, May 2002.

[25] S. M. Alamouti and S. Kallel, "Adaptive trellis-coded multiple-phasedshift keying Rayleigh fading channels," IEEE Trans. Commun., vol. 42, pp. 2305-2341, June 1994.

[26] A. J. Goldsmith and S. Chua, "Adaptive coded modulation for fading channels,” IEEE Trans. Commun., vol. 46, pp. 595-602, May 1998.

[27] D. Goeckel, "Adaptive coding for fading channels using outdated fading estimates," IEEE Trans. Commun., vol. 47, pp. 844-855, June 1999.

[28] V. Lau and M. Macleod, "Variable-rate adaptive trellis coded QAM for flat-fading channels," IEEE Trans. Commun., vol. 49, pp. 1550-1560, Sept. 2001.

[29] L. Hanzo, B. L Leap, and T. H. Liew, Turbo Coding, Turbo Equalization and Space-Time Coding. New York: Wiley, 2002.

[30] V. Tarokh, N. Seshadri, and A. R. Calderbank, "Space-time codes for high data rate wireless communication: Performance criterion and code construction," IEEE Trans. Inform. Theory, vol. 44, pp. 744-765, Mar. 1998.

[31] A. F. Naguib, V. Tarokh, N. Seshadri, and A. R. Calderbank, "A spacetime coding modem for high-data-rate wireless communications," IEEE J. Select. Areas Commun., vol. 16, pp. 1459-1478, Oct. 1998.

[32] A. Naguib, N. Seshdri, and A. Calderbank, "Increasing data rate over wireless channels: Space-time coding for high data rate wireless communications," IEEE Signal Processing Mag., vol. 17, pp. 76-92, May 2000.

[33] S. M. Alamouti, "A simple transmit diversity technique for wireless communications," IEEE J. Select. Areas Commun., vol. 16, pp. 1451-1458, Oct. 1998. 
[34] V. Tarokh, H. Jafarkhani, and A. R. Calderbank, "Space-time block coding for wireless communications: Performance results," IEEE $J$. Select. Areas Commun., vol. 17, pp. 451-460, Mar. 1999.

[35] V. Tarokh, A. Naguib, N. Seshadri, and A. R. Calderbank, "Space-time codes for high data rate wireless communication: Performance criteria in the presence of channel estimation errors, mobility, and multile paths," IEEE Trans. Commun., vol. 47, pp. 199-207, Feb. 1999.

[36] G. Bauch, A. Naguib, and N. Seshadri, "MAP equalization of spacetime coded signals over frequency selective channels," in Proc. Wireless Communications and Networking Conf., New Orleans, LA, Sept. 1999.

[37] L. Hanzo, W. Webb, and T. Keller, Single- and Multi-Carrier Quadrature Amplitude Modulation: Principles and Applications for Personal Communications, WLAN's and Broadcasting. Piscataway, NJ: IEEE Press, 2000

[38] D. Agrawal, V. Tarokh, A. Naguib, and N. Seshadri, "Space-time coded OFDM for high data-rate wireless communication over wideband channels," in Proc. IEEE Vehicular Technology Conf., Ottawa, Canada, May 1998, pp. 2232-2236.

[39] Y. Li, N. Seshadri, and S. Ariyavisitakul, "Channel estimation for OFDM systems with transmitter diversity in mobile wireless channels," IEEE J. Select. Areas Commun., vol. 17, pp. 461-471, March 1999.

[40] Y. Li, J. Chuang, and N. Sollenberger, "Transmitter diversity for OFDM systems and its impact on high-rate data wireless networks," IEEE $J$. Select. Areas Commun., vol. 17, pp. 1233-1243, July 1999.

[41] W. Choi and J. Cioffi, "Space-time block codes over frequency selective fading channels," in Proc. VTC, Amsterdam, The Netherlands, Sept. 19-22, 1999, pp. 2541-2545.

[42] A. Duel-Hallen, "Equalizers for multiple input/multiple output channels and PAM systems with cyclostationary input sequences," IEEE J. Select. Areas Commun., vol. 10, pp. 630-639, Apr. 1992.

[43] C. Tidestav, M. Sternad, and A. Ahlén, "Reuse within a cell-Interference rejection or multiuser detection," IEEE Trans. Commun., vol. 47, pp. 1511-1522, Oct. 1999

[44] T. Liew and L. Hanzo, "Space-time block coded adaptive modulation aided OFDM," in Proc. IEEE Globecom 2001, San Antonio, TX, Nov. 25-29, 2001, pp. 136-140.

[45] J. Cheung and R. Steele, "Soft-decision feedback equalizer for continuous-phase modulated signals in wide-band mobile radio channels," IEEE Trans. Commun., vol. 42, pp. 1628-1638, Feb./Mar./Apr. 1994.

[46] C. Berrou and A. Glavieux, "Near optimum error correcting coding and decoding: Turbo codes," IEEE Trans. Commun., vol. 44, pp. 1261-1271, Oct. 1996.

[47] J. Hagenauer, E. Offer, and L. Papke, "Iterative decoding of binary block and convolutional codes," IEEE Trans. Inform. Theory, vol. 42, pp. 429-445, Mar. 1996.

[48] C. E. Shannon, "A mathematical theory of communication," Bell Syst. Tech. J., pp. 379-427, 1948.

[49] O. F. Açikel and W. E. Ryan, "Punctured turbo-codes for BPSK/QPSK channels," IEEE Trans. Commun., vol. 47, pp. 1315-1323, Sept. 1999.

[50] T. Liew, J. Pliquett, B. Yeap, L. L. Yang, and L. Hanzo, "Comparative study of space time block codes and various concatenated turbo coding schemes," in Proc. PIMRC 2000, London, U.K., Sept. 18-21, 2000, pp. $741-745$

[51] T. Liew and L. Hanzo, "Space-time codes and concatenated channel codes for wireless communications," Proc. IEEE, vol. 90, pp. 183-219, Feb. 2002.

[52] L. Hanzo, F. Somerville, and J. Woodard, Voice Compression and Communications: Principles and Applications for Fixed and Wireless Channels. New York: IEEE Press, 2002.

[53] L. Hanzo, P. Cherriman, and J. Streit, Wireless Video Communications: From Second to Third Generation Systems, WLAN's and Beyond. Piscataway, NJ: IEEE Press, 2001

[54] Office for official publications of the European communities, COST207: Digital land mobile radio communications, Final Rep., Luxembourg, Belgium, 1989.

[55] J. Torrance and L. Hanzo, "Demodulation level selection in adaptive modulation," Electron. Lett., vol. 32, pp. 1751-1752, Sept. 12, 1996.

[56] J. Torrance and L. Hanzo, "On the upper bound performance of adaptive QAM in a slow Rayleigh fading," IEE Electron. Lett., pp. 169-171, Apr. 1996.

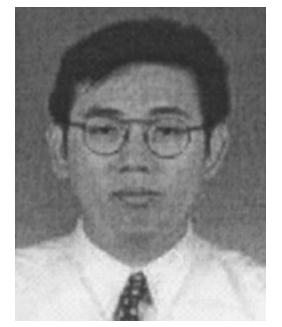

T. H. Liew received the B.Eng. degree in electronics engineering and Ph.D. degree from the University of Southampton, Southampton, U.K.

Currently, he is a Postdoctoral Research Fellow at the University of Southampton. His research interests include coding and modulation for wireless channels, space-time coding, and adaptive transceivers. He has published about 30 research papers and a research monograph

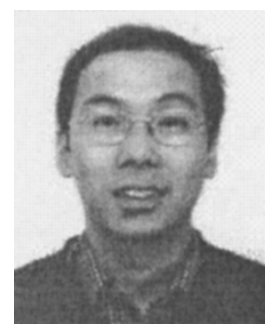

Bee Leong Yeap received the B.S. degree (first class honors) in electronics engineering and Ph.D. degree from the University of Southampton, Southampton, U.K., in 1996 and 2000, respectively.

$\mathrm{He}$ is currently a Postdoctoral Research Fellow at the University of Southampton. His research interests include turbo coding, turbo equalization, adaptive modulation, and space-time coding. He has published in excess of 12 research papers and a research monograph.

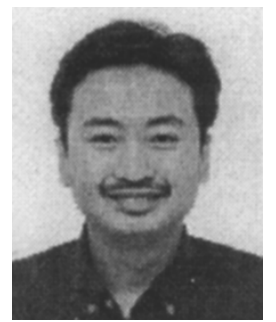

Choong Hin Wong received the B.Eng. (first class honors) degree in electronic engineering and the $\mathrm{Ph} . \mathrm{D}$. degree from the University of Southampton, Southampton, U.K. in 1995 and 1999, respectively.

His research focused mainly on equalization techniques and adaptive rate transmission schemes in a cellular mobile environment. He has published a total of ten research papers in various conferences and journals. In March 2000, he joined Multiple Access Communications Ltd., U.K., and has worked on various software development projects for the third-generation cellular mobile systems. He has published in excess of 12 research papers and a research monograph.

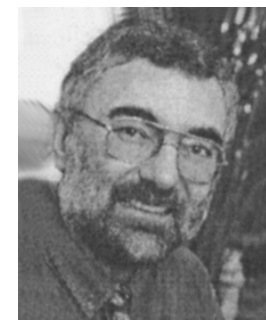

Lajos Hanzo (M'91-SM'92-F'01) received the M.S. degree in electronics, in 1976, the Ph.D. degree, in 1983, and the D.Sc. degree in 2004.

During his career in telecommunications, he has held various research and academic posts in Hungary, Germany, and the U.K. Since 1986, he has been with the Department of Electronics and Computer Science, University of Southampton, Southampton, U.K., where he holds the chair in telecommunications. He has coauthored ten books on mobile radio communications, has published in excess of 500 research papers, and has organized and chaired conference sessions, presented overview lectures, and has been awarded a number of distinctions. Currently, he heads an academic research team working on a range of research projects in the field of wireless multimedia communications sponsored by industry, the Engineering and Physical Sciences Research Council (EPSRC), U.K., the European IST Programme, and the Mobile Virtual Centre of Excellence (VCE), U.K.

Dr. Hanzo is also an IEEE Distinguished Lecturer of both the Communications as well as the Vehicular Technology Society and a Fellow of the Royal Academy of Engineering. 